\title{
El consumo de tabaco en el alumnado español pre-adolescente y adolescente: diferencias de género
}

\author{
Ramón Mendoza'; Pilar López Pérez \\ Catedrático de Psicología Evolutiva y de la Educación, Departamento de Psicología, Universidad de Huelva. \\ 2. Jefa de la Sección de Programas de Salud, Delegación Provincial de Salud (Córdoba), Consejería de Salud de la Junta de Andalucía. \\ Enviar correspondencia a: \\ Ramón Mendoza Berjano. Departamento de Psicología. Universidad de Huelva. Campus del Carmen. 21071 Huelva. España. \\ Fax: (+34) 959 219357. Correo electrónico: ramon@uhu.es.
}

Recibido: Marzo de 2007

Aceptado: Junio de 2007

\section{RESUMEN}

Este trabajo tiene como propósito describir la prevalencia del consumo de tabaco entre los pre-adolescentes y adolescentes escolarizados en España a comienzos del siglo XXI.

Los datos han sido recogidos en el marco del estudio "Estilos de Vida de los Adolescentes Escolarizados" (EVAE), una investigación de ámbito estatal de diseño transversal. La muestra es aleatoria y está compuesta por 8.429 alumnos de 10 a 18 años (49,9\% chicos y 50,1\% chicas). Se ha utilizado un cuestionario anónimo cumplimentado por los alumnos en sus propias aulas.

En el alumnado de 12 años una proporción significativamente mayor de alumnos varones que de alumnas ha fumado alguna vez tabaco. En el alumnado de 14 o más años, más chicas que chicos han fumado alguna vez tabaco. Entre los 12 y los 14 años se incrementa en 40 puntos el porcentaje de alumnas que han consumido experimentalmente tabaco.

Las tasas de fumadores diarios superan el $10 \%$ en ambos géneros a partir de los 14 años, con cifras significativamente mayores entre las chicas que entre los chicos en los grupos de edad de 15 ó más años. En el alumnado de 17 años, el 25\% de los chicos y el $35 \%$ de las chicas se manifiestan fumadores diarios.

La experiencia histórica reciente de España y de otros países muestra que es posible reducir significativamente en pocos años la prevalencia del consumo de tabaco entre los escolares. La prevención primaria del tabaquismo adolescente puede ser realmente efectiva y debe constituir una prioridad para el sistema sanitario, el sistema educativo y los otros sectores implicados.

Palabras clave: Consumo de tabaco, diferencias de género, adolescentes, escolares, España.

\section{ABSTRACT}

This paper aims to describe the prevalence of tobacco consumption among pre-adolescent and adolescent school children in Spain at the start of the 21st century.

The data were collected within the framework of the "Estilos de Vida de los Adolescentes Escolarizados" (EVAE) project, a nationwide cross-sectional study on the lifestyles of adolescent school children. In this study, a random sample of 8,429 students aged from 10 to 18 years old $(49.9 \%$ boys and $50.1 \%$ girls) was selected. The school children filled in an anonymous questionnaire in their classrooms.

Among the 12-year-old age group, there are a significantly higher number of boys than girls who have ever smoked tobacco. Figures are higher for girls in the 14-year-old or older age groups. Between the 12 and the 14-year-old age groups, there is an increase of 40 percentage points for girls who have ever smoked tobacco.

The prevalence of daily smoking exceeds $10 \%$ among the 14-year-old or older age groups, with significantly higher rates for girls than for boys among the 15-year-old group and older students. Among the 17 -year-old group, $25 \%$ of boys and $35 \%$ of girls report that they smoke daily.

The recent experience of Spain and other countries shows that it is possible to significantly reduce the prevalence of tobacco consumption among school children within a few years. The primary prevention of tobacco consumption among adolescents can be highly effective and should constitute a priority for the health system, the education system and other sectors involved.

Key words: Tobacco consumption, gender differences, adolescents, school children, Spain. 


\section{INTRODUCCIÓN}

$\mathrm{E}$ ste trabajo pretende describir cuál es la situación del consumo de tabaco entre los pre-adolescentes y adolescentes españoles a comienzos del siglo XXI. Su principal propósito es analizar la progresiva implantación del tabaquismo en la población escolarizada de 10 a 18 años, prestando especial atención a las diferencias de género en el consumo de tabaco en estas edades.

A mitad de los años ochenta comenzaron en España los estudios de ámbito estatal sobre los estilos de vida de los escolares, incluyendo el consumo de tabaco y de otras drogas (Mendoza, 1987; Mendoza, Blanco, Martín de la Concha, Morales, Ruiz, Sagrera et al., 1988). En conjunto, a lo largo de las dos últimas décadas se ha constatado una relativa estabilización de la prevalencia del tabaquismo entre los chicos y una expansión del problema entre las chicas, que ya presentan tasas de consumo más altas que los chicos (Mendoza, López y Sagrera, 2007). Ello significa una modificación de la situación tradicional de las diferencias de género en el tabaquismo adolescente en España.

El tabaquismo entre las chicas españolas era prácticamente inexistente antes de los años sesenta y continuó siendo mucho más prevalente entre los chicos que entre las chicas hasta los ochenta, según una reciente estimación retrospectiva de las tendencias en la iniciación en el consumo de tabaco en España en la segunda mitad del siglo XX (Schiaffino, Fernández, Borrel, Saltó, García y Borrás, 2003).

En nuestro contexto social, el tabaquismo es la drogodependencia con mayor impacto en la mortalidad. En la Europa occidental el tabaquismo ocasionó más de 675.000 muertes en el año 2000 (cuatro veces más muertes que el alcohol y las drogas ilegales juntos). Se ha estimado que en el año 2001 más de 49.000 personas murieron en España por enfermedades debidas al consumo de tabaco (Montes, Pérez y Gestal, 2004). En los países desarrollados la mayoría de las muertes provocadas por el tabaquismo tienen lugar en edades intermedias (entre los 35 y 69 años), conllevando un promedio de 22 años de vida perdidos (Peto, López, Boreham, Thun y Heath, 1994).

Además del impacto en la mortalidad y en la morbilidad, el tabaquismo tiene graves consecuencias sociales, económicas, educativas y ambientales, que, por lo general, han sido menos estudiadas que las consecuencias estrictamente sanitarias. Piénsese, por ejemplo, en el peso del tabaquismo en la generación de discapacidad, de dependencia y de necesidad de cuidados asistenciales, o bien en su contribución a la deforestación por incendios ocasionales en los países cálidos. En otro orden de cosas, téngase en cuenta el impacto familiar que supone que un padre o que una madre fallezcan con menos de 50 años por una enfermedad derivada del consumo de tabaco y lo que ello implica de reducción de ingresos, de pérdida de apoyo emocional a los hijos y al cónyuge, así como de pérdida de transmisión cultural y de experiencias vitales a los hijos, en una sociedad crecientemente compleja y donde la transición a la adultez continúa dilatándose.

Desde una perspectiva preventiva tiene particular sentido estudiar el tabaquismo adolescente -tanto si se piensa en términos de prevención primaria como de prevención secundaria-, básicamente por tres razones. En primer lugar, en nuestro contexto social el tabaquismo tiende a instaurarse y consolidarse mayoritariamente en la adolescencia (Infante y RubioColavida, 2004; Observatorio Español sobre Drogas, 2005; Schiaffino et al., 2003). En segundo lugar, analizar la evolución de la epidemia tabáquica entre los adolescentes nos ayuda a una mejor comprensión de su etiología y a la priorización de las medidas preventivas. La tercera razón es que la epidemia tabáquica está actualmente muy extendida entre los adolescentes -hecho que conocemos precisamente por lo que se viene investigando al respecto- y no hay fundamento para prestar atención sólo al tabaquismo adulto, cuando ya la adicción está mucho más instaurada y comienzan a manifestarse sus consecuencias a largo plazo. Aunque la experiencia de muchos países muestra que es posible reducir significativamente la prevalencia del tabaquismo entre los adultos, tiene aún más sentido desarrollar de manera sistemática programas dirigidos específicamente a los adolescentes.

Estos programas, si pretenden ser efectivos, inevitablemente han de tener en cuenta las diferencias de género que en la actualidad se observan en el consumo de tabaco en la adolescencia, ya que tanto la prevención primaria como la prevención secundaria de la epidemia pueden requerir estrategias parcialmente distintas para los chicos y para las chicas. En definitiva, el consumo de tabaco no es una conducta aislada, sino que se enmarca en unos estilos de vida que en la actualidad presentan notables diferencias de género en los adolescentes españoles (Mendoza, Batista y Rubio, 2005). Este trabajo pretende, precisamente, profundizar en el conocimiento de las diferencias de género en el consumo de tabaco en la adolescencia, así como hacer una estimación de la dimensión del problema, edad por edad, en el alumnado de 10 a 18 años del conjunto de España. El abarcar este rango de edades tan amplio permite tener una visión global de la implantación del tabaquismo entre los pre-adolescentes y adolescentes españoles, aunque circunscribiéndonos a la población escolarizada.

En concreto, los objetivos que se pretenden alcanzar son los siguientes:

1) Describir la prevalencia del consumo experimental de tabaco (haber fumado alguna vez en la vida) y 
del consumo actual (sea éste diario u ocasional) en la población escolarizada de 10 a 18 años. En cada uno de estos aspectos del consumo de tabaco se analizarán las diferencias en función de la edad, así como las diferencias en función del género (tanto las globales como dentro de cada grupo de edad).

2) Investigar si en la actualidad el hábito tabáquico presenta una mayor intensidad en la frecuencia de consumo en un género que en otro, dentro de la adolescencia.

\section{MÉTODO}

\section{Características generales del diseño}

Los datos utilizados en este trabajo han sido recogidos en el marco del estudio "Estilos de Vida de los Adolescentes Escolarizados" (EVAE). En esta investigación, de ámbito estatal y con un diseño transversal, se han estudiado los estilos de vida, la autopercepción, las relaciones sociales, la información sobre el SIDA y determinadas conductas de protección y de riesgo del alumnado de 10 a 18 años.

La metodología de este proyecto es muy similar a la de la serie de estudios "Health Behaviour in School-aged Children" (HBSC, o ECERS, en sus siglas en castellano) Ilevados a cabo entre 1986 y 1994 en nuestro país (Mendoza, Sagrera y Batista, 1994), que a su vez comparten características metodológicas básicas con el estudio internacional HBSC, auspiciado por la OMS (Aaro, Wold, Kannas y Rimpelä, 1986; Mendoza, Batista y Oliva, 1994). En particular, la metodología del EVAE coincide con la de los estudios HBSC-ECERS en lo referente al sistema de muestreo, el tipo de instrumento y su forma de administración. El cuestionario del EVAE contiene algunas preguntas comunes con las de estos estudios, pero otras son específicas de este nuevo proyecto.

\section{Sujetos}

La muestra del estudio EVAE ha sido diseñada para que sea representativa del alumnado pre-adolescente y adolescente de todo el Estado. Para ello, se han elegido aleatoriamente 204 centros docentes de todo el país y, dentro de ellos, dos aulas, también de manera aleatoria. La muestra obtenida ha sido de 8.956 sujetos.

El diseño de la muestra ha sido estratificado (por cursos y áreas geográficas), proporcional -cada municipio y cada centro docente se encontraba proporcionalmente representado en función de su censo-, aleatorio y polietápico (se eligen al azar municipios, centros y aulas sucesivamente). Se ha pretendido asegurar que todos los alumnos de 10 a 18 años matriculados en algún centro docente del país tengan la misma probabilidad de salir elegidos en la muestra. En un informe anterior se ha descrito el sistema de muestreo pormenorizadamente (Mendoza, et al., 1994b). El 92,2\% de los centros seleccionados accedieron gustosamente a colaborar. Los que declinaron la invitación a participar en el estudio fueron aleatoriamente sustituidos por otros del mismo estrato y del mismo tipo de titularidad (pública o privada).

Se ha llevado a cabo el trabajo de campo entre septiembre y diciembre de 2002. En conjunto, en el día en que se realizó la encuesta en cada centro asistieron a clase el $92 \%$ de los alumnos matriculados. De ellos, rehusaron cumplimentar el cuestionario el $0,5 \%$, lo que equivale a un promedio de un alumno cada ocho aulas encuestadas. Aproximadamente la mitad de dichos alumnos alegaron motivos ajenos al contenido del cuestionario (como ser inmigrante y no conocer suficientemente el idioma).

En este trabajo se analizan únicamente los datos relativos a los sujetos con edades comprendidas entre los 10 y los 18 años (8.429 alumnos; 49,9\% chicos y $50,1 \%$ chicas). La tabla 1 contiene el desglose de esta muestra por edad y sexo.

Tabla 1. Distribución de la muestra del estudio EVAE por edad y sexo.

\begin{tabular}{|c|c|c|c|c|c|c|c|c|c|c|}
\cline { 2 - 11 } \multicolumn{1}{c|}{} & $\begin{array}{c}\mathbf{1 0} \\
\text { años }\end{array}$ & $\begin{array}{c}\mathbf{1 1} \\
\text { años }\end{array}$ & $\begin{array}{c}\mathbf{1 2} \\
\text { años }\end{array}$ & $\begin{array}{c}\mathbf{1 3} \\
\text { años }\end{array}$ & $\begin{array}{c}\mathbf{1 4} \\
\text { años }\end{array}$ & $\begin{array}{c}\mathbf{1 5} \\
\text { años }\end{array}$ & $\begin{array}{c}\mathbf{1 6} \\
\text { años }\end{array}$ & $\begin{array}{c}\mathbf{1 7} \\
\text { años }\end{array}$ & $\begin{array}{c}\mathbf{1 8} \\
\text { años }\end{array}$ & $\begin{array}{c}\text { Total } \\
\text { Chicos }\end{array}$ \\
\hline \multirow{2}{*}{ Chicas } & 502 & 522 & 577 & 496 & 505 & 533 & 467 & 400 & 205 & 4207 \\
& $43.2 \%$ & $51.8 \%$ & $50.3 \%$ & $51.2 \%$ & $50.6 \%$ & $47.8 \%$ & $48.0 \%$ & $45.6 \%$ & $51.1 \%$ & $49.9 \%$ \\
\hline \multirow{2}{*}{ Total } & $46.8 \%$ & $48.2 \%$ & $49.7 \%$ & $48.8 \%$ & $49.4 \%$ & $52.2 \%$ & $52.0 \%$ & $54.4 \%$ & $48.9 \%$ & $50.1 \%$ \\
& $100 \%$ & $100 \%$ & $100 \%$ & $100 \%$ & $100 \%$ & $100 \%$ & $100 \%$ & $100 \%$ & $100 \%$ & $100 \%$ \\
\hline
\end{tabular}




\section{Instrumento, variables y proceso de recogida de datos}

Los datos han sido recogidos mediante un cuestionario anónimo, cumplimentado por los alumnos en su propia aula en presencia de un encuestador profesional ajeno al centro docente. Una vez terminado el cuestionario, cada alumno lo ha introducido en un sobre y lo ha cerrado. Los sobres han sido remitidos inmediatamente al centro coordinador del estudio (Universidad de Huelva). El tiempo medio empleado en responder al cuestionario ha sido 32 minutos.

El cuestionario está muy focalizado en la recogida de información sobre los estilos de vida, pero también recaba datos sobre otros aspectos de interés para el planteamiento del estudio (véase Mendoza et al., 2005, para mayor detalle).

Su elaboración ha requerido varios estudios previos (entrevistas grupales con adolescentes y un pre-piloto), así como un estudio piloto en primavera de 2002, llevado a cabo con una muestra de 886 alumnos de cinco comunidades autónomas. Parte de los ítems utilizados provienen de los estudios HBSC-ECERS, como ya se ha indicado, por lo que habían sido probados con anterioridad en varias ocasiones.

Se han preparado tres modelos del cuestionario, en función de la edad de los alumnos, con un núcleo de preguntas comunes a todos ellos. De cada uno de estos modelos se han preparado versiones equivalentes en cuatro lenguas (castellano, catalán, gallego y vasco). La equivalencia de estas versiones se ha verificado por un proceso de retraducción, así como en el estudio piloto.

La mayor parte de las preguntas se presentan de forma cerrada. En las contadas ocasiones en las que los sujetos tienen que escribir las respuestas con sus propias palabras, éstas han sido posteriormente codificadas con criterios homogéneos. Todos los modelos del cuestionario incluyen una presentación inicial del estudio y varias preguntas finales de valoración del instrumento.

En aproximadamente la mitad de las aulas (51\%) ha estado presente algún representante del equipo docente (un profesor, en el 73\% de esos casos, o bien el tutor o el director). Se ha procurado que la presencia de estas personas no interfiriese el clima de confidencialidad del estudio.

Este trabajo está focalizado en la información proveniente de dos preguntas relativas al consumo de tabaco. Ambas cuestiones han estado presentes en todos los modelos del cuestionario. La primera se refiere al consumo experimental (haber fumado alguna vez tabaco en la vida) y la segunda, al consumo actual (en el periodo de administración del cuestionario).

En concreto, la primera cuestión está formulada en los siguientes términos: “¿Has fumado tabaco, aunque sólo sea una vez? (al menos, un cigarrillo, un puro o una pipa).". Se plantean a continuación dos posibles respuestas: "Sí" y "No". La segunda cuestión es: "¿Con qué frecuencia fumas actualmente?". La gama de respuestas es la siguiente: "Todos los días", "Una vez a la semana o más, pero no cada día", "Menos de una vez por semana" y "No fumo".

Estas preguntas no han planteado consultas de los alumnos a los encuestadores. Ambas cuestiones han originado una tasa muy baja de omisiones (en general, menor del $2 \%$ ), excepto en los grupos de 10 y 11 años, donde son algo mayores (véanse las tablas 2 y 3 ).

\section{Estrategia de análisis de los datos}

Se ha efectuado un análisis bivariado de cada una de las dos variables objeto de estudio (consumo experimental de tabaco y consumo actual) en función del género y de la edad. En cada casilla de las tablas de contingencia resultantes se ha calculado el residuo ajustado estandarizado, que es una estimación de la diferencia entre la frecuencia observada en dicha casilla y la frecuencia esperable si las dos variables fueran completamente independientes. Este valor normalizado equivale a la clásica Z cuando se comparan dos proporciones observadas. Denota una diferencia significativa entre la frecuencia observada y la frecuencia esperada cuando es igual o superior a 1,96, si se adopta como criterio un riesgo alfa del $5 \%$. Los resultados de estos análisis están reflejados en las tablas 2 y 3 , donde además se indica la precisión de cada una de las estimaciones realizadas en relación con estas dos variables. Los porcentajes están calculados tomando como base (100\%) a los sujetos que responden cada pregunta.

\section{RESULTADOS}

\section{Experimentación del consumo de tabaco}

En ambos géneros, la proporción de alumnos que han fumado alguna vez tabaco es directamente proporcional a la edad. En el alumnado de 10 años, cifras muy similares de alumnos varones y de alumnas manifiestan haber experimentado el consu- 
mo de tabaco $(6,4 \%$ y 4,7\%, respectivamente). En el alumnado de 18 años, las tasas son mucho más altas $y$, a su vez, significativamente mayor la de las chicas $(88,8 \%)$ que la de los chicos $(77,5 \%)$. Entre los alumnos varones, cuando se comparan las tasas de consumo experimental entre los distintos grupos de edad, el incremento más notable en función de la edad a este respecto se observa entre los 13 y los 14 años (30,7\% y 45,3\%, respectivamente). En lo que se refiere a las chicas, el segmento de edad con un mayor incremento es más precoz: 12-13 años, donde se pasa del $13 \%$ al 34,8\%, es decir, más de 20 puntos de diferencia. Sin embargo, las alumnas presentan también otro segmento de edad con un incremento llamativo: el de 13-14 años, donde se detectan casi 20 puntos de diferencia $(34,8 \%$ y $53,3 \%)$. En conjunto, entre los 12 y los 14 años se incrementa en 40 puntos el porcentaje de alumnas que han fumado alguna vez tabaco (figura 1 y tabla 2).

En lo que se refiere a las diferencias de género en la tasa de experimentación con el tabaco, dentro de cada grupo de edad, se constata que en el alumnado de 12 años una proporción significativamente mayor de alumnos varones que de alumnas ha fumado alguna vez tabaco. La situación se invierte en el alumnado de 13 años, pero las diferencias sólo son significativas desde los 14 años en adelante. En conjunto, pues, en lo que respecta al alumnado de 14 ó más años, se observa que más chicas que chicos han fumado alguna vez tabaco.

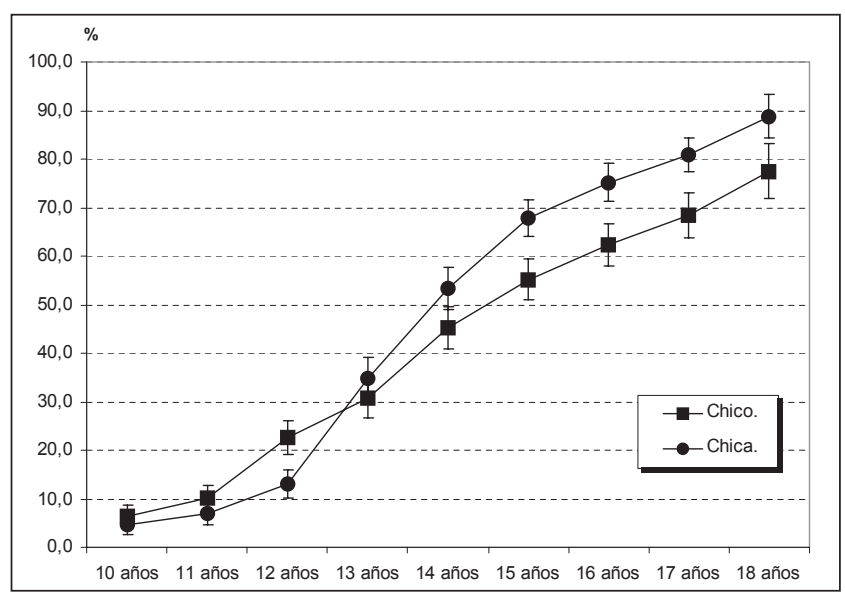

Figura 1. Estudiantes de 10 a 18 años que han probado tabaco, según el género (España, 2002).

\section{Consumo diario de tabaco}

Las tasas de fumadores diarios son cercanas al 0\% entre el alumnado de 10 u 11 años. En el de 12 años, rondan el $1 \%$ en ambos géneros. Superan el
$10 \%$ a partir de los 14 años, con cifras significativamente mayores entre las chicas que entre los chicos en todos los grupos de edad. En el alumnado de 18 años, un tercio de los chicos (33\%) y aproximadamente la mitad de las chicas $(53,8 \%)$ se manifiestan fumadores diarios (figura 2 y tabla 3 ).

Entre los chicos, cuando se comparan las tasas de consumo diario entre unos grupos de edad y otros, el incremento de la proporción de fumadores diarios en función de la edad es relativamente constante a partir de los 13 años. En cambio, entre las chicas se constatan incrementos notables en tres segmentos de edad: 13-14 años, 14-15 años y, sobre todo, 17-18 años. Así, si en el grupo de edad de 13 años son fumadoras diarias "sólo" el 3\% de las alumnas, en el de 15 años la cuarta parte de las alumnas $(24,7 \%)$ manifiestan consumir cotidianamente tabaco. A su vez, el incremento en las respectivas tasas entre los 17 y los 18 años es casi de 20 puntos $(34,8 \%$ y $53,8 \%$, respectivamente).

En cuanto a las diferencias de género en la tasa de consumo diario de tabaco dentro de cada grupo de edad, éstas no son significativas en los grupos de edad menores de 14 años. En el grupo de 14 años las chicas presentan un porcentaje mayor que los chicos, pero la diferencia sólo roza la significación estadística $(z=1,9)$. En cambio, en todos los grupos de mayor edad (15 años en adelante) las chicas presentan prevalencias de consumo diario notablemente superiores a las de los chicos (tabla 3).

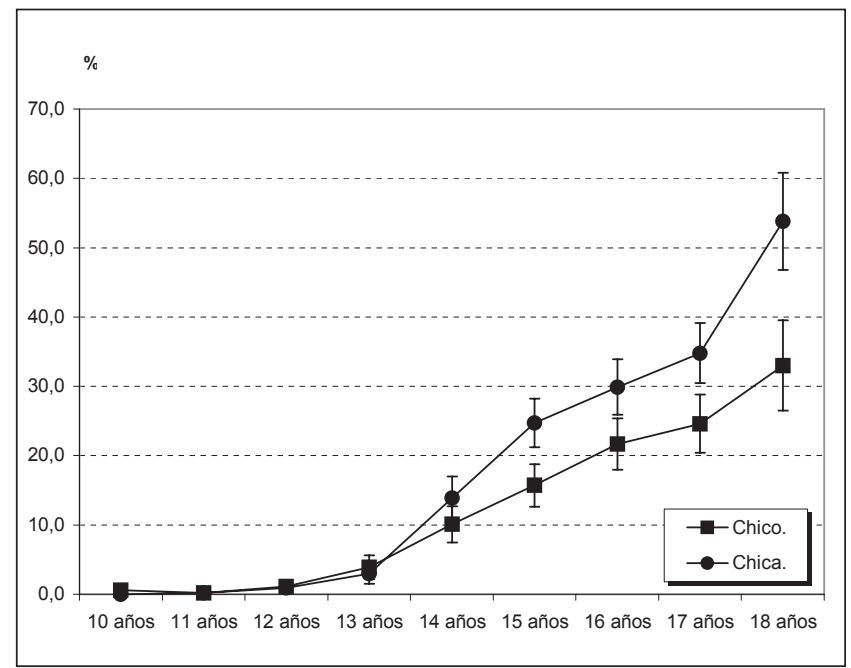

Figura 2. Prevalencia del consumo diario de tabaco entre el alumnado de 10 a 18 años, según el género (España, 2002). 


\section{Consumo ocasional}

Ambos géneros presentan patrones relativamente similares en lo que se refiere a la evolución de las tasas de consumo ocasional en función de la edad. Por consumo ocasional se entiende aquí el que no es diario, bien tenga una periodicidad al menos semanal o bien sea más esporádico.

En lo que se refiere a los alumnos varones, el consumo de tabaco es básicamente ocasional hasta los 13 años de edad, aunque a dicha edad la tasa de fumadores diarios no es despreciable (3,9\%). En los grupos de edad de 14 años y superiores, una proporción relativamente similar de alumnos (en torno al $12 \%$ ) se manifiestan fumadores ocasionales (figura 3 y tabla 3). Nótese que ello no significa que alrededor del $12 \%$ de los alumnos varones se mantengan como fumadores ocasionales (no diarios) a partir de los 14 años de edad, ya que el estudio es transversal y no longitudinal -se están comparando grupos de edad, no viendo la evolución de una determinada cohorte-. Dentro de este $12 \%$ aproximado de alumnos varones que fuman ocasionalmente en todos los grupos de edad (a partir de los 14 años), tienen un peso relativamente similar los fumadores regulares no diarios -fuman al menos una vez a la semana-y los fumadores más esporádicos.

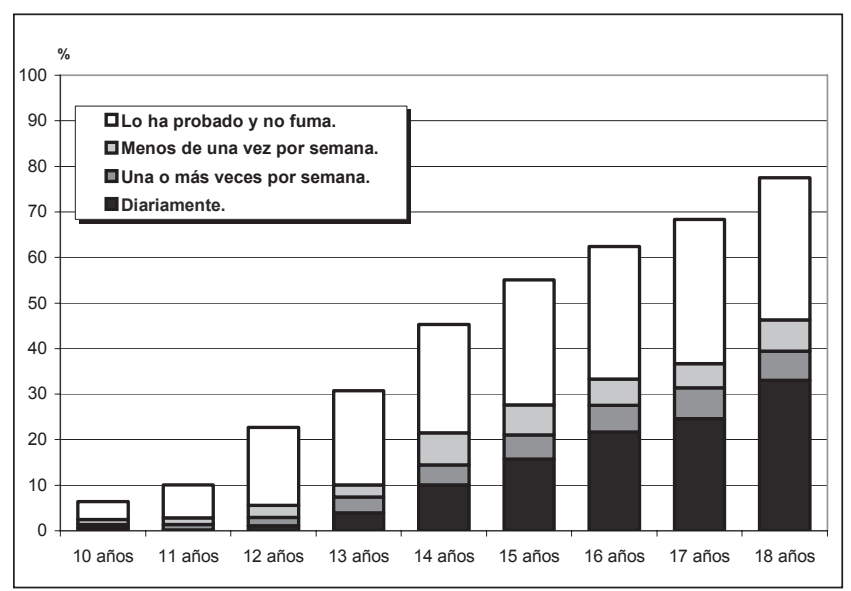

Figura 3. Distribución de la frecuencia de consumo de tabaco entre los chicos escolarizados, según la edad (España, 2002).

En lo que se refiere a las alumnas, al igual que entre los alumnos varones, el porcentaje de fumadoras diarias es inferior al de fumadoras ocasionales en los grupos de edad 10 a 13 años. A su vez, la tasa de fumadoras ocasionales ronda entre el 11 y el $14 \%$ en los grupos de edad de 13 a 17 años, también con un peso relativamente similar de las fumadoras regulares y de las fumadoras más esporádicas. El grupo de 18 años muestra dos peculiaridades: una tasa muy alta de fumadoras diarias, ya comentada $(53,8 \%)$, y una tasa casi inexistente de fumadoras esporádicas con una frecuencia menor a la semanal $(0,5 \%)$. La tasa de fumadoras regulares no diarias en este grupo de edad es del $8,7 \%$ (figura 4 y tabla 3 ). Expresado en otros términos, entre las chicas escolarizadas de 18 años casi todas las fumadoras son diarias y, las que no lo son, fuman al menos una vez a la semana.

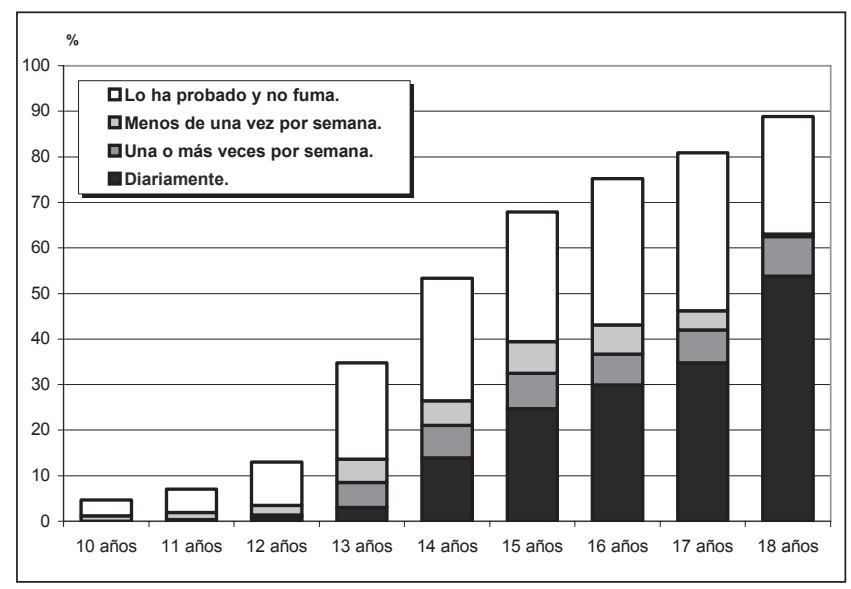

Figura 4. Distribución de la frecuencia de consumo de tabaco entre las chicas escolarizadas, según la edad (España, 2002).

En cuanto a las diferencias de género en la prevalencia del consumo regular no diario (fumar por lo menos una vez a la semana), los alumnos varones tienen tasas más altas que las alumnas en los grupos de edad inferiores a 13 años, pero la diferencia es significativa sólo en el grupo de 12 años. En los grupos de edad de 13 o más años, los porcentajes de las chicas a este respecto son más algo más altos que los de los chicos, pero las diferencias no llegan a ser significativas en ninguno de estos grupos de edad. Así, pues, en conjunto puede afirmarse que no se detectan notables diferencias de género en lo que se refiere a la prevalencia del consumo regular no diario.

En cuanto al consumo más esporádico, la situación de las diferencias de género es parecida. Sólo se detectan diferencias significativas en el grupo de edad de 18 años, con una tasa notablemente mayor entre los chi$\cos (6,9 \%)$ que entre las chicas $(0,5 \%)$ (tabla 3$)$. 
Tabla 2. Experimentación del consumo de tabaco entre el alumnado de 10 a 18 años, según el género (España, 2002).

\begin{tabular}{|c|c|c|c|c|c|c|}
\hline \multirow[b]{2}{*}{ Edad } & \multirow[b]{2}{*}{ Categoría } & \multicolumn{2}{|c|}{ Chicos } & \multicolumn{2}{|c|}{ Chicas } & \multirow{2}{*}{$\begin{array}{c}\text { Significación } \\
\text { (Z) }\end{array}$} \\
\hline & & $\%$ & $\begin{array}{l}\text { Margen error } \\
\text { (IC 95\%) }\end{array}$ & $\%$ & $\begin{array}{l}\text { Margen error } \\
\text { (IC 95\%) }\end{array}$ & \\
\hline \multirow{3}{*}{10 años } & Ha probado tabaco & 6,4 & $\pm 2,2$ & 4,7 & $\pm 2,0$ & 1,1 \\
\hline & No lo ha probado & 93,6 & $\pm 2,2$ & 95,3 & $\pm 2,0$ & 1,1 \\
\hline & Sujetos / TNR & \multicolumn{2}{|c|}{$\mathrm{n}=502(\mathrm{TNR}=3,4 \%)$} & \multicolumn{2}{|c|}{$n=442(\operatorname{TNR}=4,3 \%)$} & \\
\hline \multirow{3}{*}{11 años } & Ha probado tabaco & 10,1 & $\pm 2,6$ & 7,0 & $\pm 2,3$ & 1,7 \\
\hline & No lo ha probado & 89,9 & $\pm 2,6$ & 93,0 & $\pm 2,3$ & 1,7 \\
\hline & Sujetos / TNR & \multicolumn{2}{|c|}{$n=522($ TNR $=3,1 \%)$} & \multicolumn{2}{|c|}{$n=485(T N R=2,5 \%)$} & \\
\hline \multirow{3}{*}{12 años } & Ha probado tabaco & 22,7 & $\pm 3,5$ & 13,0 & $\pm 2,8$ & 4,3 \\
\hline & No lo ha probado & 77,3 & $\pm 3,5$ & 87,0 & $\pm 2,8$ & 4,3 \\
\hline & Sujetos / TNR & \multicolumn{2}{|c|}{$\mathrm{n}=577(\mathrm{TNR}=3,1 \%)$} & \multicolumn{2}{|c|}{$n=570(T N R=1,4 \%)$} & \\
\hline \multirow{3}{*}{13 años } & Ha probado tabaco & 30,7 & $\pm 4,1$ & 34,8 & $\pm 4,3$ & 1,4 \\
\hline & No lo ha probado & 69,3 & $\pm 4,1$ & 65,2 & $\pm 4,3$ & 1,4 \\
\hline & Sujetos / TNR & \multicolumn{2}{|c|}{$n=496($ TNR $=1,4 \%)$} & \multicolumn{2}{|c|}{$\mathrm{n}=473(\mathrm{TNR}=0,4 \%)$} & \\
\hline \multirow{3}{*}{14 años } & Ha probado tabaco & 45,3 & $\pm 4,4$ & 53,3 & $\pm 4,4$ & 2,5 \\
\hline & No lo ha probado & 54,7 & $\pm 4,4$ & 46,7 & $\pm 4,4$ & 2,5 \\
\hline & Sujetos / TNR & \multicolumn{2}{|c|}{$n=505(T N R=1,2 \%)$} & \multicolumn{2}{|c|}{$\mathrm{n}=493(\mathrm{TNR}=0 \%)$} & \\
\hline \multirow{3}{*}{15 años } & Ha probado tabaco & 55,1 & $\pm 4,2$ & 67,9 & $\pm 3,8$ & 4,4 \\
\hline & No lo ha probado & 44,9 & $\pm 4,2$ & 32,1 & $\pm 3,8$ & 4,4 \\
\hline & Sujetos / TNR & \multicolumn{2}{|c|}{$n=533(T N R=0,6 \%)$} & \multicolumn{2}{|c|}{$\mathrm{n}=581 \quad(\mathrm{TNR}=0,2 \%)$} & \\
\hline \multirow{3}{*}{16 años } & Ha probado tabaco & 62,4 & $\pm 4,4$ & 75,2 & $\pm 3,8$ & 4,3 \\
\hline & No lo ha probado & 37,6 & $\pm 4,4$ & 24,8 & $\pm 3,8$ & 4,3 \\
\hline & Sujetos / TNR & \multicolumn{2}{|c|}{$n=467(T N R=0,2 \%)$} & \multicolumn{2}{|c|}{$n=505(T N R=0,2 \%)$} & \\
\hline \multirow{3}{*}{17 años } & Ha probado tabaco & 68,3 & $\pm 4,6$ & 80,9 & $\pm 3,5$ & 4,3 \\
\hline & No lo ha probado & 31,7 & $\pm 4,6$ & 19,1 & $\pm 3,5$ & 4,3 \\
\hline & Sujetos / TNR & \multicolumn{2}{|c|}{$\mathrm{n}=400(\mathrm{TNR}=0,8 \%)$} & \multicolumn{2}{|c|}{$\mathrm{n}=477(\mathrm{TNR}=0,2 \%)$} & \\
\hline \multirow{3}{*}{18 años } & Ha probado tabaco & 77,5 & $\pm 5,7$ & 88,8 & $\pm 4,4$ & 3,0 \\
\hline & No lo ha probado & 22,5 & $\pm 5,7$ & 11,2 & $\pm 4,4$ & 3,0 \\
\hline & Sujetos / TNR & \multicolumn{2}{|c|}{$\mathrm{n}=205(\mathrm{TNR}=0,5 \%)$} & $n=$ & $J R=0 \%)$ & \\
\hline
\end{tabular}

TNR = Tasa de no respuesta (omisiones en la pregunta) 
Tabla 3. Consumo de tabaco entre el alumnado de 10 a 18 años, según el género (España, 2002).

\begin{tabular}{|c|c|c|c|c|c|c|}
\hline \multirow[b]{2}{*}{ Edad } & \multirow[b]{2}{*}{ Categoría } & \multicolumn{2}{|c|}{ Chicos } & \multicolumn{2}{|c|}{ Chicas } & \multirow{2}{*}{$\begin{array}{c}\text { Significación } \\
\text { (Z) }\end{array}$} \\
\hline & & $\%$ & $\begin{array}{l}\text { Margen error } \\
\text { (IC 95\%) }\end{array}$ & $\%$ & $\begin{array}{l}\text { Margen error } \\
\text { (IC 95\%) }\end{array}$ & \\
\hline \multirow{5}{*}{10 años } & Fuma diariamente & 0,6 & $\pm 0,7$ & 0,0 & $\pm 0,0$ & 1,6 \\
\hline & 1 ó más veces/sem. & 0,8 & $\pm 0,8$ & 0,0 & $\pm 0,0$ & 1,9 \\
\hline & Menos de 1 vez/sem. & 1,1 & $\pm 0,9$ & 1,2 & $\pm 1,1$ & 0,2 \\
\hline & No fuma & 97,5 & $\pm 1,4$ & 98,8 & $\pm 1,1$ & 1,4 \\
\hline & Sujetos / TNR & \multicolumn{2}{|c|}{$n=502(T N R=5,4 \%)$} & \multicolumn{2}{|c|}{$\mathrm{n}=442(\mathrm{TNR}=7,7 \%)$} & \\
\hline \multirow{5}{*}{11 años } & Fuma diariamente & 0,2 & $\pm 0,4$ & 0,2 & $\pm 0,4$ & 0,1 \\
\hline & 1 ó más veces/sem. & 1,2 & $\pm 1,0$ & 0,2 & $\pm 0,4$ & 1,8 \\
\hline & Menos de 1 vez/sem. & 1,4 & $\pm 1,0$ & 1,5 & $\pm 1,1$ & 0,2 \\
\hline & No fuma & 97,2 & $\pm 1,4$ & 98,0 & $\pm 1,3$ & 0,8 \\
\hline & Sujetos / TNR & \multicolumn{2}{|c|}{$\mathrm{n}=522(\mathrm{TNR}=4,2 \%)$} & \multicolumn{2}{|c|}{$\mathrm{n}=485(\mathrm{TNR}=5,4 \%)$} & \\
\hline \multirow{5}{*}{12 años } & Fuma diariamente & 1,1 & $\pm 0,9$ & 0,9 & $\pm 0,8$ & 0,3 \\
\hline & 1 ó más veces/sem. & 1,8 & $\pm 1,1$ & 0,5 & $\pm 0,6$ & 2,0 \\
\hline & Menos de 1 vez/sem. & 2,7 & $\pm 1,3$ & 2,1 & $\pm 1,2$ & 0,6 \\
\hline & No fuma & 94,5 & $\pm 1,9$ & 96,4 & $\pm 1,5$ & 1,6 \\
\hline & Sujetos / TNR & \multicolumn{2}{|c|}{$n=577($ TNR $=3,1 \%)$} & \multicolumn{2}{|c|}{$\mathrm{n}=570(\mathrm{TNR}=1,8 \%)$} & \\
\hline \multirow{5}{*}{13 años } & Fuma diariamente & 3,9 & $\pm 1,7$ & 3,0 & $\pm 1,5$ & 0,8 \\
\hline & 1 ó más veces/sem. & 3,5 & $\pm 1,6$ & 5,5 & $\pm 2,1$ & 1,5 \\
\hline & Menos de 1 vez/sem. & 2,7 & $\pm 1,4$ & 5,1 & $\pm 2,0$ & 1,9 \\
\hline & No fuma & 89,9 & $\pm 2,7$ & 86,4 & $\pm 3,1$ & 1,7 \\
\hline & Sujetos / TNR & \multicolumn{2}{|c|}{$\mathrm{n}=496(\mathrm{TNR}=1,8 \%)$} & \multicolumn{2}{|c|}{$\mathrm{n}=473(\mathrm{TNR}=0,4 \%)$} & \\
\hline \multirow{5}{*}{14 años } & Fuma diariamente & 10,1 & $\pm 2,6$ & 13,9 & $\pm 3,1$ & 1,9 \\
\hline & 1 ó más veces/sem. & 4,4 & $\pm 1,8$ & 7,1 & $\pm 2,3$ & 1,8 \\
\hline & Menos de 1 vez/sem. & 7,0 & $\pm 2,2$ & 5,4 & $\pm 2,0$ & 1,1 \\
\hline & No fuma & 78,5 & $\pm 3,6$ & 73,7 & $\pm 3,9$ & 1,8 \\
\hline & Sujetos / TNR & \multicolumn{2}{|c|}{$\mathrm{n}=505(\mathrm{TNR}=1,6 \%)$} & \multicolumn{2}{|c|}{$\mathrm{n}=493(\mathrm{TNR}=2,2 \%)$} & \\
\hline \multirow{5}{*}{15 años } & Fuma diariamente & 15,7 & $\pm 3,1$ & 24,7 & $\pm 3,5$ & 3,7 \\
\hline & 1 ó más veces/sem. & 5,3 & $\pm 1,9$ & 7,8 & $\pm 2,2$ & 1,6 \\
\hline & Menos de 1 vez/sem. & 6,6 & $\pm 2,1$ & 6,9 & $\pm 2,1$ & 0,2 \\
\hline & No fuma & 72,3 & $\pm 3,8$ & 60,6 & $\pm 4,0$ & 4,1 \\
\hline & Sujetos / TNR & \multicolumn{2}{|c|}{$n=533($ TNR $=1,1 \%)$} & $n=5$ & $=0,3 \%$ & \\
\hline & Fuma diariamente & 21,7 & $\pm 3,7$ & 29,9 & $\pm 4,0$ & 2,9 \\
\hline & 1 ó más veces/sem. & 5,8 & $\pm 2,1$ & 6,8 & $\pm 2,2$ & 0,6 \\
\hline 16 años & Menos de 1 vez/sem. & 5,8 & $\pm 2,1$ & 6,4 & $\pm 2,1$ & 0,4 \\
\hline & No fuma & 66,7 & $\pm 4,3$ & 57,0 & $\pm 4,3$ & 3,1 \\
\hline & Sujetos / TNR & $n=$ & $=0,4 \%$ & $n=5$ & $=0,6 \%$ & \\
\hline & Fuma diariamente & 24,6 & $\pm 4,2$ & 34,8 & $\pm 4,3$ & 3,3 \\
\hline & 1 ó más veces/sem. & 6,8 & $\pm 2,5$ & 7,2 & $\pm 2,3$ & 0,2 \\
\hline 17 años & Menos de 1 vez/sem. & 5,3 & $\pm 2,2$ & 4,2 & $\pm 1,8$ & 0,7 \\
\hline & No fuma & 63,4 & $\pm 4,7$ & 53,8 & $\pm 4,5$ & 2,9 \\
\hline & Sujetos / TNR & $n=$ & $=0,3 \%$ & $n=4$ & $=0,6 \%$ & \\
\hline & Fuma diariamente & 33,0 & $\pm 6,5$ & 53,8 & $\pm 7,0$ & 4,2 \\
\hline & 1 ó más veces/sem. & 6,4 & $\pm 3,4$ & 8,7 & $\pm 4,0$ & 0,9 \\
\hline 18 años & Menos de 1 vez/sem. & 6,9 & $\pm 3,5$ & 0,5 & $\pm 1,0$ & 3,3 \\
\hline & No fuma & 53,7 & $\pm 6,9$ & 36,9 & $\pm 6,8$ & 3,4 \\
\hline & Sujetos / TNR & $n=$ & $R=1 \%)$ & $n=1$ & $=0,5 \%$ & \\
\hline
\end{tabular}

TNR = Tasa de no respuesta (omisiones en la pregunta) 


\section{DISCUSIÓN}

\section{Aspectos metodológicos}

En primer lugar, cabe plantearse en qué medida el método utilizado permite confiar en la calidad de la información aquí presentada y si esos resultados son extrapolables al conjunto de la población adolescente española.

El sistema de muestreo del estudio EVAE, que ha sido reiteradamente utilizado en los estudios HBSC-ECERS en nuestro país, permite considerar a la muestra utilizada (de casi 9.000 sujetos) como representativa de la población escolarizada en España de 10 a 18 años. Sin embargo, no debe olvidarse que la escolaridad en nuestro país es obligatoria sólo hasta los 16 años, por lo que a partir de dicha edad no puede identificarse a la población escolarizada con la población real del país. Cualquier estudio con este diseño muestral inevitablemente deja fuera a los adolescentes de una determinada edad que no estén escolarizados. Según datos del Ministerio de Educación y Ciencia relativos al curso 2001-2002, en España está escolarizado el 100\% de la población entre 10 y 15 años, pero sólo el 87,6\% de la población de 16 años, el $72,4 \%$ de la de 17 años y el $57,6 \%$ de la de 18 años (Ministerio de Educación y Ciencia, 2004).

La calidad de la muestra viene también respaldada por el hecho de que sólo el 7,8\% de los centros elegidos aleatoriamente rehusaran colaborar y tuvieran que ser sustituidos por otros, también aleatoriamente. Esta tasa es similar a la del estudio HBSC-ECERS de 1994 (7,3\%). En cuanto a los alumnos, la cifra de alumnos absentistas el día de administración del cuestionario en sus aulas es relativamente baja ( 2 por aula, de media). Es destacable que sólo el 0,5\% de los alumnos presentes se negaran a contestar el cuestionario $(0,3 \%$ en el estudio HBSC-ECERS de 1994).

Se ha cuidado al máximo que el diseño y la presentación del instrumento, su carácter anónimo y las condiciones de aplicación favorecieran la sinceridad de los alumnos en las respuestas. Tanto el cuestionario como el sistema de administración fueron puestos a prueba en el estudio piloto. Las preguntas relativas al consumo de tabaco habían sido probadas, además, en los estudios HBSC-ECERS, sin que plantearan problemas de comprensión.

La inteligibilidad de las preguntas relativas al consumo de tabaco viene respaldada también por el hecho de que en el trabajo de campo del EVAE no han motivado consultas particulares a los encuestadores por parte de los alumnos. A su vez, la presencia de personal del centro en el $51 \%$ de las aulas en el momento de administrar el cuestionario no parece haber afectado a la sinceridad de las respuestas a estas preguntas. Este aspecto ha sido explorado en cálculos comple- mentarios a los aquí presentados. En ningún grupo de edad se ha detectado que las respuestas a las preguntas de consumo de tabaco (experimentación y consumo actual) varíen significativamente en función de la presencia o no de personal del centro en el aula. La única excepción la constituye el grupo de 11 años, en lo referente a la experimentación, con una prevalencia del 7,3\% si no hay personal del centro, $12,3 \%$ si está presente un profesor y $4,1 \%$ si está presente el tutor. Estas pequeñas diferencias, de escasa relevancia, pueden quizás interpretarse en el sentido de que algunos centros pueden haber preferido que esté presente un profesor cuando el aula elegida por azar es particularmente conflictiva y, a su vez, quizás sea en las aulas conflictivas donde más extendido esté el consumo de tabaco en esa edad.

\section{Consistencia interna de los resultados}

Una segunda cuestión que nos podemos plantear es si los resultados aquí presentados gozan de coherencia interna. Hay dos aspectos, al menos, en los que los resultados son consistentes entre sí.

En primer lugar, hay concordancia entre las diferencias de género en la prevalencia del consumo experimental de tabaco y las relativas al consumo diario. En ambos aspectos, las chicas presentan tasas significativamente más altas que los chicos en el grupo de edad de 15 años y superiores. En el grupo de 14 años se presentan también el mismo tipo de diferencias de género, aunque, como ya se ha comentado más arri$\mathrm{ba}$, en lo que se refiere al consumo diario la diferencia sólo roza la significación estadística.

En segundo lugar, parece verosímil que en ambos géneros la tasa de fumadores diarios se incremente proporcionalmente a la edad. Es sobradamente conocida la capacidad adictiva de la nicotina, por lo que parece lógico que, cuanto mayor sea la edad de los alumnos, mayor sea la proporción de fumadores diarios dentro del conjunto de los fumadores.

\section{Contraste con otros estudios}

La estructura interna de estos resultados es esencialmente similar a la de los obtenidos en los tres estudios HBSC-ECERS Ilevados a cabo en España entre 1986 y 1994 (Mendoza, 1987; Mendoza et al., 1994b; Mendoza, Batista, Sánchez y Carrasco, 1998). Así, en concreto, en este trabajo, al igual que en estos tres estudios, se ha constatado lo siguiente: 1) el consumo de tabaco tiende a hacerse más frecuente cuanto más alta es la edad de los alumnos o alumnas; 2) existe un paralelismo entre la evolución de la prevalencia del consumo experimental y la evolución de la prevalencia del consumo diario, cuando se comparan entre sí los 
distintos grupos de edad (ambas prevalencias siguen pendientes parecidas en función de la edad, pero, lógicamente, con tasas menores en lo que se refiere al consumo diario de tabaco); y 3) la tasa de alumnos fumadores ocasionales es bastante similar en todos los grupos de edad.

Ahora bien, los resultados de este trabajo aportan, por vez primera, información diferenciada sobre la prevalencia del consumo de tabaco en el alumnado de cada grupo de edad entre los 10 y los 18 años, con lo que se obtiene una visión más global y una mejor comprensión de la situación del tabaquismo en el conjunto del alumnado no universitario del país. Esta información, que se refiere a la situación a comienzos del siglo XXI (2002), presenta algunas diferencias con los hallazgos de estos estudios previos en lo relativo a los grupos de edad comunes a todos ellos (básicamente, el alumnado de 11, 13 y 15 años). Dichas diferencias resultan verosímiles y han sido analizadas pormenorizadamente en un reciente trabajo sobre las tendencias temporales en el consumo de tabaco entre los escolares españoles (Mendoza et al., 2007). El definitiva, el tabaquismo adolescente no es un fenómeno socialmente estático, sino que tiende a evolucionar en la medida en que se modifican los factores que lo determinan.

Cabe también preguntarse en qué medida los datos concuerdan con los de otros estudios de ámbito estatal que hayan recogido información sobre el consumo de tabaco entre los escolares y que hayan sido llevados a cabo en el mismo año, aunque sea en una época estacional distinta. En este sentido, los datos del EVAE relativos al consumo de tabaco pueden ser contrastados con los del estudio español HBSC y con los de la encuesta sobre consumo de drogas entre escolares del Plan Nacional sobre Drogas (PNSD).

La comparación entre los resultados del EVAE y los del HBSC español de 2002 viene facilitada por el hecho de que la metodología de ambas investigaciones entronca con la de la serie de estudios HBSC-ECERS en España y, a su vez, con la del estudio internacional HBSC. Esto hace que el método de ambos estudios sea muy similar en numerosos aspectos, incluyendo la forma de medir el consumo experimental y el consumo actual de tabaco (para mayor detalle, véase Currie, Roberts, Morgan, Smith, Settertobulte, Samdal et al., 2004; Mendoza et al., 1994b; Moreno, Muñoz, Pérez y Sánchez, 2005). En cuanto al contraste entre los resultados del EVAE y los de la encuesta del PNSD hay que tener en cuenta que la metodología de ambos estudios, aunque es sustancialmente similar, presenta también notables diferencias. En concreto, entre otros aspectos, en la encuesta del PNSD se aborda sólo a la población escolarizada de 14 a 18 años (Infante y Rubio-Colavida, 2004; Observatorio Español sobre Drogas, 2005).
A su vez, la formulación de las preguntas de consumo de tabaco difiere de las del EVAE, aunque algunas de las variables estudiadas son básicamente las mismas, lo que permite cierta comparabilidad.

Veamos, en primer lugar, lo referente a la prevalencia del consumo experimental de tabaco (haber fumado alguna vez en la vida). Aquí nos ceñiremos al contraste entre el EVAE y el HBSC español de 2002, ya que se dispone de la información desagregada por sexo y edad del HBSC, al menos respecto a los grupos de edad de 11, 13 y 15 años (Godeau, Rahav y Hublet, 2004). En lo que se refiere a las alumnas de estas edades, las prevalencias estimadas en ambos estudios son muy similares, con diferencias en los porcentajes que oscilan entre 0,2 puntos y 1,4 puntos. En cambio, en cuanto a los chicos, el HBSC-02 hace estimaciones algo más altas del consumo experimental de tabaco, en particular en el grupo de 13 años de edad (7,6 puntos de diferencia) y en el de 15 años $(4,4$ puntos de diferencia).

En segundo lugar, contrastemos la prevalencia en el consumo de tabaco de frecuencia de al menos una vez a la semana. Tanto el HBSC como el EVAE permiten calcular esta prevalencia, combinando la categoría de respuesta relativa al consumo diario y la referente al consumo de periodicidad al menos semanal, pero no diario. En este aspecto, la coincidencia en las estimaciones de ambos estudios diferenciando géneros en cada una de las tres edades mencionadas (11, 13 y 15 años) es casi total, con discrepancias que en general son menores al 1\%. Así, por ejemplo, según el EVAE el 32,5\% de las alumnas de 15 años fuman tabaco con una periodicidad al menos semanal; según el HBSC, son el 32,3\% (Godeau et al., 2004).

En tercer lugar, veamos lo referente al consumo diario. En cuanto a las edades de 11 y de 13 años, las prevalencias estimadas por el EVAE son bastante coincidentes con las del HBSC-02, con diferencias de 1,1 puntos como máximo. En la edad de 15 años disponemos de información proveniente de los tres estudios. Las estimaciones del EVAE y del HBSC son muy similares, con diferencias de 1,5 puntos como máximo; en cambio, las del estudio del PNSD son algo divergentes. En concreto, la prevalencia estimada del consumo diario de tabaco entre los chicos de 15 años es $15,7 \%$ en el EVAE, 16,6\% en el HBSC (Godeau et al., 2004) y $12,4 \%$ en el estudio del PNSD (Infante y Rubio-Colavida, 2004). En cuanto a las chicas, las cifras son $24,7 \%, 23,2 \%$ y $17,8 \%$ respectivamente.

En lo que se refiere al consumo diario de tabaco en los grupos de edad de 16, 17 y 18 años, nos ceñiremos al EVAE y al estudio del PNSD, del que se dispone de los datos desagregados por género en cada una de estas edades. Las estimaciones de las prevalencias a este respecto en ambos estudios discrepan, en general, menos de cuatro puntos y llegan a ser 
muy coincidentes en algunos subgrupos (por ejemplo, 24,6\% de chicos fumadores diarios de 17 años según el EVAE y $24 \%$ según la encuesta del PNSD). La única discrepancia relevante es la relativa a las chicas de 18 años, donde la prevalencia estimada por el EVAE es de 53,8\% y la del estudio del PNSD, 45,6\% (Infante y Rubio-Colavida, 2004). En lo que respecta a los chicos de esta edad, las estimaciones de ambos estudios son muy coincidentes $(33 \%$ y $31,1 \%$, respectivamente).

Es interesante observar que tanto en el EVAE como en el estudio del PNSD se constata un gran salto en la prevalencia del consumo diario entre las chicas de 17 años y las de 18 (en el caso del EVAE, de 19 puntos de diferencia; en el estudio del PNSD, de 14,6 puntos). También en ambos estudios se detectan en estos grupos de edad tasas de consumo diario notablemente superiores entre las chicas que entre los chicos. Así, por ejemplo, en el grupo de 16 años las dos tasas se diferencian en 8,2 puntos según el EVAE y en 9,4 puntos según la encuesta del PNSD; en el de 18 años, las diferencias son de 20,8 puntos y 14,5 respectivamente.

Los resultados de estos tres estudios de ámbito estatal se ciñen a la población escolarizada de unas determinadas edades. En el estudio "Salud y juventud", llevado a cabo con una muestra de jóvenes de 13 a 29 años (escolarizados o no) de siete comunidades autónomas, también se ha detectado una mayor prevalencia del consumo de tabaco entre las chicas que entre los chicos, pero exclusivamente en el hábitat urbano, no en el rural (Hernán, Ramos y Fernández, 2002). Por su parte, el Informe 2004 Juventud en España no presenta los datos de consumo de tabaco provenientes de las encuestas de 2001 y 2003 desagregados por edad y género, lo que impide su contraste con los resultados aquí presentados. En todo caso, es interesante constatar que los resultados de la encuesta del Instituto de la Juventud de 2001 muestran que la prevalencia del tabaquismo entre los jóvenes españoles es directamente proporcional a la edad. En concreto, la tasa de fumadores (de ambos sexos) varía desde el 15,8\% en el grupo de 15 años hasta el $52 \%$ a los 29 años. Por otra parte, globalmente se ha detectado en estos estudios un incremento del tabaquismo en los jóvenes españoles entre 2001 y 2003 (36,1\% de prevalencia global del consumo de tabaco en 2001 entre los jóvenes de 15 a 29 años y 42,6\% en 2003) (Comas, 2005).

\section{La expansión de la epidemia tabáquica entre las adolescentes}

El tabaquismo entre las mujeres es un fenómeno relativamente reciente en nuestro país. Aunque se ha detectado una prevalencia creciente del consumo de tabaco entre las mujeres españolas desde los años cincuenta, la tasa de fumadoras diarias no comenzó a superar el 10\% hasta 1975. Continuó creciendo hasta alcanzar el 22,5\% en 1995 (Fernández, Schiaffino, García, Saltó, Villalbí y Borrás, 2003). A partir de 1995, las estimaciones de la Encuesta Nacional de Salud muestran una estabilización (en torno al 25\%) de la tasa de mujeres fumadoras diarias (Infante y RubioColavida, 2004).

El tabaquismo adolescente, como fenómeno colectivo, anticipa el tabaquismo adulto en una sociedad, ya que previsiblemente una buena parte de los fumadores adolescentes (de uno u otro género) mantendrán su hábito hasta la adultez, a no ser que en esa sociedad se desarrollen de manera sistemática y con una orientación adecuada programas de prevención primaria y de prevención secundaria del tabaquismo. Por ello, la actual difusión del tabaquismo entre las chicas españolas escolarizadas hace prever un futuro próximo en el que tasas más altas que en la actualidad de mujeres de edad media serán fumadoras de tabaco, lo que previsiblemente incrementará en nuestra comunidad los problemas de salud ligados al tabaquismo en la mujer.

El Consejo Asesor de Tabaquismo de Cataluña ha efectuado una amplia revisión sobre el impacto del tabaquismo en la salud de la mujer, que en algunos aspectos tiene características comunes con las consecuencias sanitarias de este hábito para los hombres, pero en otros tiene su propia especificidad. Así, al igual que le ocurre a los hombres, las mujeres fumadoras tienen un riesgo especial de sufrir determinados tipos de cánceres (de pulmón, de la cavidad oral, de faringe, de laringe, de esófago, de vejiga y de páncreas, entre otros). En cuanto a las complicaciones cardiovasculares, aunque la mayoría de las acciones del humo del tabaco sobre el aparato circulatorio no son específicas del género, el riesgo de sufrir una enfermedad coronaria es superior en el caso de una mujer fumadora. Lo mismo se puede decir la asociación del hábito tabáquico con la diabetes. A su vez, las mujeres que toman anticonceptivos orales tienen un riesgo 20 veces mayor de infarto de miocardio, en especial si tienen más de 35 años. Se ha constatado que el hábito tabáquico ejerce un papel negativo sobre el efecto protector que tienen los estrógenos respecto al riesgo cardiovascular en la mujer. Así mismo, se ha constatado que el tabaquismo favorece la menopausia precoz, aumenta el riesgo de osteoporosis en la perimenopausia, aumenta el riesgo de embarazo extrauterino, dificulta la implantación del embrión en el endometrio y aumenta el riesgo de infertilidad, entre otras alteraciones. No hay que olvidar, por otra parte, que se han descrito preocupantes efectos del consumo de tabaco en la gestación y en la infancia, como son el retardo en el crecimiento intrauterino y el bajo peso al nacer, así como un mayor riesgo de 
aborto espontáneo, ruptura prematura de membranas, parto prematuro, síndrome de muerte súbita del lactante, infecciones respiratorias y asma infantil (Salleras, 2001).

Históricamente en la generalidad de las sociedades se ha disuadido, de diversas formas, el consumo de tabaco y de alcohol entre las jóvenes. A medida que la industria tabaquera ha puesto de manera creciente a la mujer como punto de mira de sus campañas promocionales, el consumo de tabaco entre este sector de la población ha ido creciendo. Amos y Haglund (2000) distinguen dos etapas básicas en la sofisticada estrategia que la industria tabaquera ha seguido durante el siglo XX para promover el consumo de tabaco entre las mujeres: 1) acabar con el rechazo social hacia el consumo de tabaco en la mujer; 2) convertir el consumo de tabaco en un símbolo de emancipación, independencia y éxito social de la mujer. Así, consumir tabaco pasa de ser una conducta socialmente estigmatizada a convertirse en una práctica socialmente aceptable e incluso deseable. Como consecuencia de esta estrategia, durante el siglo XX la epidemia se expandió primero entre las mujeres de Norte América y del norte de Europa y después entre las del sur de Europa. Actualmente se detecta una fuerte expansión del problema entre las mujeres de países sometidos a intensas transformaciones sociales, donde la industria tabaquera continúa presentando el consumo de tabaco como un pasaporte hacia la emancipación, la independencia y el éxito. Según este trabajo, éste es el caso de los países del este de Europa y de numerosos países en vías de desarrollo.

La promoción del tabaquismo entre las mujeres por parte de la industria tabaquera no se ha restringido a la publicidad del tabaco. Ha incluido también la creación de marcas especialmente dirigidas a las mujeres y el patrocinio de famosas cantantes, entre otras actividades (Amos, 1996). A su vez, la prensa femenina en Europa con frecuencia ha dado una visión positiva del consumo de tabaco por la mujer, en particular en sus imágenes (Amos, Bostock y Bostock, 1998).

En nuestro país, la expansión del tabaquismo entre las mujeres es fruto de factores específicos como los arriba descritos, pero también obedece a factores generales que han contribuido a expandir el tabaquismo en el conjunto de la sociedad, en especial en la segunda mitad del siglo XX (Mendoza, 1980)

Cualquier forma de promoción del consumo de tabaco en el conjunto de la población acaba repercutiendo en los adolescentes, ya que éstos son muy sensibles a lo que socialmente se presenta como prestigiado. Además, hay factores que probablemente han afectado de manera particular a los adolescentes de ambos géneros. Entre ellos, los siguientes: la frecuente inclusión de protagonistas jóvenes en los anuncios de tabaco (Montes y Alvarez, 2006), la presencia de vallas anunciando tabaco en las cercanías de los colegios, la inserción de anuncios de este producto en la prensa universitaria, la retransmisión televisa de competiciones de vehículos de motor con una fuerte presencia de anuncios de tabaco, la conversión de las cajetillas de cigarrillos en soportes publicitarios, la alta disponibilidad de dinero de bolsillo por parte de los adolescentes (Ariza-Cardenal y Nebot-Adell, 2002; Mendoza et al., 1994b), la facilidad que tienen niños y pre-adolescentes para adquirir tabaco en los establecimientos de venta (Luque, Mesas, Alberro, Palma, Ortiz y Cáliz et al., 2002) y la existencia de una amplia red de máquinas de venta automática de tabaco, que a su vez ha servido de soporte de atractivos anuncios. A este respecto, es interesante constatar que un reciente estudio experimental llevado a cabo en Australia ha comprobado que la publicidad del tabaco en los puntos de venta crea en los niños un sentido de familiaridad con este producto, lo que puede predisponerlos a su consumo (Wakefield, Germain, Durkin y Henriksen, 2006). No hay que olvidar, por otra parte, las dificultades que en nuestro país encuentran muchos docentes para sistematizar la práctica de la educación para la salud en su actividad cotidiana en las aulas.

La prohibición de la publicidad del tabaco en la nueva legislación española (desde enero de 2006) conllevará previsiblemente un cambio significativo en esta situación. Lamentablemente, la industria tabaquera ha intensificado la publicidad del tabaco en los primeros años de este siglo en los medios de comunicación escritos (Montes y Alvarez, 2006), lo que muy probablemente ha contribuido a expandir la epidemia entre las chicas y a que no se reduzca entre los chicos.

Un factor que puede haber contribuido de manera indirecta, pero significativa, a la expansión y el mantenimiento del tabaquismo entre las adolescentes y las mujeres en general es el hecho de que exista una fuerte presión social para imponer la extrema delgadez como el canon estético deseable de belleza femenina, en combinación con la creencia de que fumar tabaco ayuda a "guardar la línea". Se ha constatado que, entre las chicas, la insatisfacción con el propio cuerpo está estrechamente asociada con el consumo de tabaco (Mendoza et al., 2005). La industria tabaquera, por su parte, ha elaborado anuncios presentando el fumar como un medio para adelgazar (Amos, 1996).

\section{Sugerencias para la intervención preventiva}

La prevención del tabaquismo adolescente requiere la combinación de medidas genéricas de reduc- 
ción de la oferta y de la demanda de tabaco con otras dirigidas más específicamente a este sector de la población.

Ante una epidemia tan grave en sus consecuencias y tan extendida -recuérdese que aproximadamente la mitad de las chicas escolarizadas de 18 años se manifiestan fumadoras diarias, así como un tercio de los chicos de esa edad- es necesario dinamizar programas de intervención (bien orientados) en cualquier entorno donde convivan los adolescentes o desde donde se pueda acceder a ellos. Así, aunque los centros docentes son un entorno privilegiado de actuación en este terreno, existen otros ámbitos en donde es posible y deseable poner en marcha programas de prevención primaria y secundaria del tabaquismo (servicios de atención a la juventud, centros sanitarios, servicios sociales, centros de trabajo, barrios, asociaciones y espacios de ocio, entre otros).

Los programas de prevención primaria del tabaquismo en la pre-adolescencia y en la adolescencia deben proponerse, como objetivos básicos, reducir la incidencia del consumo experimental de tabaco y la del consumo ocasional, y no sólo tratar de reducir la incidencia del consumo diario. Sin duda, es el consumo diario el que conlleva las consecuencias más graves para la salud, pero no hay que olvidar que fumar diariamente es el final de un proceso que, entre sus pasos previos, tuvo la experimentación y el consumo ocasional. Existe evidencia de que el consumo ocasional es un fuerte predictor del consumo diario (Braverman, Svendsen, Lund y Aaro, 2001). A su vez, tanto los resultados aquí presentados como los de la serie de estudios HBSC-ECERS muestran un claro paralelismo entre las tasas de prevalencia del consumo experimental y las tasas de prevalencia del consumo diario, cuando se analizan en función de la edad, como ya se ha comentado. De ahí se puede colegir que es muy improbable que se pueda reducir significativamente la incidencia del consumo diario si antes no se han puesto en marcha, de manera idónea, iniciativas tendentes a reducir la experimentación con este producto adictivo y el paso de dicha experimentación al consumo ocasional.

Desde esta perspectiva, conviene prestar especial atención a los segmentos de edad en los que se detectan incrementos más notables en las tasas de experimentación (12-13 años en los chicos y 12-14 años en las chicas). El final de la educación primaria y el primer ciclo de la educación secundaria se manifiestan así como las etapas más críticas en lo que se refiere a la prevención del consumo experimental de tabaco.

En cuanto al consumo diario, se ha detectado un incremento de más de 20 puntos en su prevalencia entre las alumnas de 13 años y las de 15, así como otro incremento de magnitud parecida entre las chi- cas de 17 años y las de 18. Entre los chicos, como se ha visto, el incremento de esta tasa en función de la edad es más homogéneo. Estos datos deberían ser tenidos en cuenta en la planificación de programas de prevención primaria tendentes a reducir la incidencia del consumo diario, así como en los de prevención secundaria que pretendan estimular y facilitar el abandono temprano de este hábito adictivo.

Por otra parte, no interesa olvidar que el sector del alumnado con peor adaptación escolar es el que presenta tasas mayores de consumo de tabaco (Mendoza et al., 1994b). Si se pretende ser efectivos, el diseño de los programas y su desarrollo han de tener en cuenta este hecho. Igualmente, no se puede soslayar que un sector relevante de los fumadores pre-adolescentes y adolescentes no anticipan que acabarán adictos a la nicotina (Rugkåsa, Stewart-Knox, Sittlington, Santos Abaunza y Treacy, 2003), lo que también debe ser tenido en cuenta a la hora de seleccionar los contenidos informativos de los programas dirigidos a estos sectores de la población.

La experiencia histórica reciente de nuestro país -y la de otros- muestra que es posible reducir significativamente en pocos años la prevalencia del consumo de tabaco entre los escolares (Mendoza et al., 2007). La prevención primaria del tabaquismo adolescente puede ser realmente efectiva y debe constituir una prioridad para el sistema sanitario, el sistema educativo y los otros sectores implicados.

Ahora bien, la prevención secundaria de este problema también tiene especial sentido, sobre todo teniendo en cuenta que un sector de los adolescentes está intentando dejar de fumar y parte de ellos afirma haberlo conseguido con éxito. En el HBSCECERS de 1990 se detectó que el 55\% del alumnado que consumía tabaco en dicho año afirmaba haber intentando alguna vez dejar de fumar (Mendoza et al., 1994b). Más recientemente, en el estudio "Salud y juventud" se ha constatado que el $27 \%$ de los adolescentes entre 13 y 17 años y el 39\% de los jóvenes entre 18 y 29 años afirman haber intentado dejar de fumar. De aquéllos que lo han intentado, un tercio lo ha conseguido. La mayoría de los que lo han conseguido opinan que lo lograron sin ayuda (Hernán et al., 2002). El contexto es, pues, relativamente favorable a la puesta en marcha de programas destinados a estimular el abandono temprano de esta adicción. Un diseño cuidadoso de estos programas y su evaluación posterior ayudarán a ir delimitando cuáles son los enfoques más efectivos de intervención a este respecto en nuestro contexto sociocultural.

Si en numerosos países la epidemia tabáquica se está expandiendo entre las mujeres, parece imprescindible abogar por un reconocimiento generalizado, en todos los ámbitos, de que el tabaquismo femenino constituye un problema sanitario de primer orden 
(Amos y Haglund, 2000). A su vez, en la intervención preventiva puede ser necesario combinar programas dirigidos a los adolescentes de ambos géneros con otros más específicamente orientados hacia la prevención del tabaquismo entre las chicas o las mujeres jóvenes en general.

La magnitud de la epidemia, la gravedad de sus consecuencias y su vulnerabilidad como problema de salud justifican que las instituciones prioricen la intervención en este terreno. En todo caso, no debe olvidarse que la inversión económica que requiere la prevención del tabaquismo es notablemente inferior al presupuesto que se está empleando en la asistencia a los afectados por sus consecuencias.

\section{AGRADECIMIENTOS}

La realización del estudio "Estilos de Vida de los Adolescentes Escolarizados" (EVAE) ha sido posible gracias al apoyo económico de FIPSE (1999-2003) y a la colaboración institucional de las consejerías de educación de todas las comunidades autónomas, junto con la participación de los 204 centros docentes elegidos aleatoriamente.

\section{REFERENCIAS}

Aaro, L. E., Wold, B., Kannas, L., Rimpelä, M. (1986). Health behaviour in schoolchildren: a WHO cross-national survey: a presentation of philosophy, methods and selected results of the first survey. Health Promotion, 1, 17-33.

Amos, A. (1996). Women and smoking. British Medical Bulletin, 52, 74-89.

Amos, A., Bostock, C., Bostock, Y. (1998). Women's magazines and tobacco in Europe. Lancet, 352, 786-787.

Amos, A., Haglund, M. (2000). From social taboo to "torch of freedom": the marketing of cigarettes to women. Tobacco Control, 9, 3-8.

Ariza-Cardenal, C., Nebot-Adell, M. (2002). Factors associated with smoking progression among Spanish adolescents. Health Education Research, 17, 750-760.

Braverman, M. T., Svendsen, T., Lund, K. E., Aaro, L. E. (2001). Tobacco use by early adolescents in Norway. European Journal of Public Health, 11, 218-224.

Currie, C., Roberts, C., Morgan, A., Smith, R., Settertobulte, W., Samdal, O. et al. (Eds.) (2004). Young people's health in context: Health Behaviour in School-aged Children (HBSC) study: international report from the 2001/2002 survey. Copenhagen: World Health Organization.

Fernández, E., Schiaffino, A., García, M., Saltó, E., Villalbí, J. R. y Borrás, J.M. (2003). Prevalencia del consumo de tabaco en España entre 1945 y 1995. Reconstrucción a partir de las Encuestas Nacionales de Salud. Medicina Clínica (Barcelona), 120, 24-26.

Godeau, E., Rahav, G., Hublet, A. (2004). Tobacco smoking. En: Currie, C., Roberts, C., Morgan, A., Smith, R., Settertobulte, W., Samdal, O. et al. (Eds.). Young people's health in context: Health Behaviour in Schoolaged Children (HBSC) study: international report from the 2001/2002 survey (pp. 63-72). Copenhagen: World Health Organization.

Comas, D. (2005). Las experiencias de la vida: aprendizajes y riesgos. En: Aguinaga, J., Andréu, J., Cachón, L., Comas, D., López, A. y Navarrete, L. Informe 2004 Juventud en España (pp. 277-478). Madrid: Instituto de la Juventud.

Hernán, M., Ramos, M. y Fernández, A. (2002). Salud y juventud. Madrid: Consejo de la Juventud de España.

Infante, C. y Rubio-Colavida, J. M. (2004). La prevalencia del consumo de tabaco en España. Adicciones, 16 (Supl. 2), 59-73.

Luque, M. J., Mesas, A., Alberro, J., Palma, S., Ortiz, F. y Cáliz, A. et al., (2002). ¿Qué piensan los niños sobre el tabaco? Medicina de Familia. Andalucía, 3, 184-189.

Mendoza, R. (1980). El tabac com a droga contaminant: epidemiologia del tabaquisme al nostre país. En: Xlè Congrés de Metges i Biòlegs de Llengua Catalana: $2^{a}$ ponència: Ecologia i Salut (pp. 229-239). Barcelona.

Mendoza, R. (1987). Consumo de tabaco y alcohol en los escolares españoles. Comunidad y Drogas, 5/6, 83-102.

Mendoza, R., Batista, J. M., Sánchez, M., Carrasco, A (1998). El consumo de tabaco, alcohol y otras drogas en los adolescentes escolarizados españoles. Gaceta Sanitaria, 12, 263-271.

Mendoza, R., Batista, J. M., Oliva, A. (1994a). Life-styles of European school-children: findings of the WHO cross national study on health-related behaviour. En: Dauwalder, J.P. (Ed.) Psychology and promotion of health (pp. 8-20). Bern: Hogrefe \& Huber Publishers.

Mendoza, R., Batista, J. M., Rubio, A. (2005). El desarrollo de estilos de vida en los adolescentes escolarizados: diferencias entre chicos y chicas. Revista de Psicología General y Aplicada, 58, 51-74.

Mendoza, R., Blanco, J., Martín de la Concha, P., Morales, E., Ruiz, J., Sagrera, R. et al. (1988). Los escolares y la salud: estudio de los hábitos de los escolares españoles en relación con la salud. Madrid: Ministerio de Sanidad y Consumo.

Mendoza, R., López, P., Sagrera, M. R (2007). Diferencias de género en la evolución del tabaquismo adolescente en España (1986-2002) Adicciones 19, 273-287.

Mendoza, R., Sagrera, M. R., Batista, J. M. (1994b). Conductas de los escolares españoles relacionadas con la salud (1986-1990). Madrid: Consejo Superior de Investigaciones Científicas.

Ministerio de Educación y Ciencia (2004). Las cifras de la educación en España: Estadísticas e indicadores: Edición 2004. Madrid: Secretaría General Técnica del Ministerio de Educación y Ciencia. 
Montes, J. y Alvarez, M. L. (2006). Tabaco y medios de comunicación escritos en España: una atracción fatal. Gaceta Sanitaria, 20, 59-62.

Montes Martínez, A., Pérez Ríos, M. y Gestal Otero, J. J. (2004). Impacto del tabaquismo sobre la mortalidad en España. Adicciones, 16 (Supl. 2), 75-81.

Moreno, M. C., Muñoz, M. V., Pérez, P. y Sánchez, I. (2005). Los adolescentes españoles y su salud: Resumen del estudio Health Behaviour in School Aged Children (HBSC-2002). Madrid: Ministerio de Sanidad y Consumo.

Observatorio Español sobre Drogas (2005). Informe 2004. Madrid: Delegación del Gobierno para el Plan Nacional sobre Drogas, Ministerio de Sanidad y Consumo.

Peto, R., López, A. D., Boreham, J., Thun, M., Heath, C. (1994). Mortality from smoking in developed countries 1950-2000. Oxford: Oxford University Press.

Rugkåsa, J., Stewart-Knox, B., Sittlington, J., Santos Abaunza, P., Treacy, M. P. (2003). Hard boys, attractive girls: expressions of gender in young people's conversations on smoking in Northern Ireland. Health Promotion International, 18, 307-314.

Salleras, L. (Ed.) (2001). Dona i tabac: Informe tècnic sobre I'hàbit tabàquic femení a Catalunya: Informe del Consell Assessor sobre Tabaquisme a Catalunya. Barcelona: Generalitat de Catalunya, Departament de Sanitat i Seguretat Social.

Schiaffino, A., Fernández, E., Borrel, C., Saltó, E., García, M. y Borrás, J.M. (2003). Gender and educational differences in smoking initiation rates in Spain from 1948 to 1992. European Journal of Public Health, 13, 56-60.

Wakefield, M., Germain, D., Durkin, S., Henriksen, L. (2006). An experimental study of effects on schoolchildren of exposure to point-of-sale cigarette advertising and pack displays. Health Education Research, 21, 338-347. 
